\title{
Shear viscosity of nuclear matter
}

\author{
A. G. Magner* \\ Institute for Nuclear Research NASU, 03680 Kiev, Ukraine \\ M. I. Gorenstein \\ Bogolyubov Institute for Theoretical Physics NASU, 03680, Kiev, Ukraine \\ U. V. Grygoriev and V. A. Plujko \\ Taras Shevchenko National University, 03022 Kiev, Ukraine
}

(Dated: October, 9th 2016)

\begin{abstract}
Shear viscosity $\eta$ is calculated for the nuclear matter described as a system of interacting nucleons with the van der Waals (VDW) equation of state. The Boltzmann-Vlasov kinetic equation is solved in terms of the plane waves of the collective overdamped motion. In the frequent-collision regime, the shear viscosity depends on the particle-number density $n$ through the mean-field parameter $a$, which describes attractive forces in the VDW equation. In the temperature region $T=15-40 \mathrm{MeV}$, a ratio of the shear viscosity to the entropy density $s$ is smaller than 1 at the nucleon number density $n=(0.5-1.5) n_{0}$, where $n_{0}=0.16 \mathrm{fm}^{-3}$ is the particle density of equilibrium nuclear matter at zero temperature. A minimum of the $\eta / s$ ratio takes place somewhere in a vicinity of the critical point of the VDW system. Large values of $\eta / s \gg 1$ are, however, found in both the low-density, $n \ll n_{0}$, and high-density, $n>2 n_{0}$, regions. This makes the ideal hydrodynamic approach inapplicable for these densities.
\end{abstract}

PACS numbers: 21.10.Ev, 21.60.Cs, 24.10.Pa

Keywords: shear viscosity, nuclear matter, van der Waals equation of state

\section{INTRODUCTION}

The shear viscosity $\eta$ and its ratio to the entropy density $s$ became recently attractive (see, e.g., Refs. [1-4] and references therein) in connection with a development of the hydrodynamic approach to the relativistic nucleusnucleus collisions. Chapman and Enskog (CE) obtained [5-10] the shear viscosity $\eta$ in a gas of non-relativistic particles by using the Boltzmann kinetic equation (BKE) for the phase-space distribution function $f(\mathbf{r}, \mathbf{p}, t)$, where $\mathbf{r}$ and $\mathbf{p}$ are the particle coordinate and momentum, respectively, and $t$ denotes the time variable.

The BKE was solved within the frequent-collision (FC) regime for which one can use a perturbation expansion in a small parameter, e.g., $\omega / \nu$, where $\nu$ is the collision frequency and $\omega$ measures the characteristic dynamical variations of the distribution function $\delta f(\mathbf{r}, \mathbf{p}, t)$. In this case the Boltzmann integral collision term is dominant as compared to other collisionless terms. For their calculations the local-equilibrium distribution function $f_{\text {l.e. }}$ was used in a standard form in terms of the evolution of particle-number density $n(\mathbf{r}, t)$, temperature $T(\mathbf{r}, t)$, and collective velocity $\mathbf{u}(\mathbf{r}, t)$. The hydrodynamic variables $n(\mathbf{r}, t)$ and $\mathbf{u}(\mathbf{r}, t)$ are defined as the zero and first moments of the distribution function in the momentum space. Thus, the evolution derivative of the distribution function, $\mathrm{d} f / \mathrm{d} t$, as one of the local equilibrium distribution, $\mathrm{d} f_{\text {1.e. }} / \mathrm{d} t$, in solving the BKE at the first order in $\omega / \nu$ can be decomposed into terms proportional to that of $n(\mathbf{r}, t), T(\mathbf{r}, t)$, and $\mathbf{u}(\mathbf{r}, t)$. Using then the standard closed system of hydrodynamical equations and condition $\delta T=0$, one obtains $[5,8-10]$ the expression for the shear viscosity $\eta$. For a gas of elastic scattering balls with the diameter $d$, at the first approximation in $\omega / \nu$, one finds [5]

$$
\eta_{\mathrm{CE}}=\frac{5}{16 \sqrt{\pi}} \frac{\sqrt{m T}}{d^{2}}
$$

where $m$ is the particle mass. The shear viscosity $\eta_{\mathrm{CE}}$ appears to be independent of the particle-number density $n$ because, at the first order in small parameter $\omega / \nu$, the attractive interaction on large distances between particles was neglected to simplify the $\mathrm{CE}$ viscosity calculations.

Extensions of the $\mathrm{CE}$ method to the relativistic high energy-density problems are given in Refs. [3, 11]. In particular, Eq. (1) was reproduced in the nonrelativistic limit within the CE approach in Ref. [3]. The mixture of different hadron species was considered in Ref. [12]. Several investigations were devoted to go beyond the hydrodynamical approach [5]; see, e.g., Refs. [13-28]. In contrast to the $\mathrm{CE}$ approach, the main problem solved in these works was to take into account a self-consistent mean field in calculations of the viscosity of Fermi liquids within the Landau quasiparticle theory.

In the present paper, we use the Boltzmann-Vlasov kinetic equation (BVKE) for a system of interacting nucleons with the van der Waals (VDW) equation of state. Therefore, both scattering of particles owing to the hardcore repulsions and Vlasov self-consistent mean field, owing to the VDW attractive interaction, are taken into account in solving the BVKE.

In our consideration, the small dynamical variations $\delta f$ are found from a linearized BVKE in the simplified form,

$$
\delta f(\mathbf{r}, \mathbf{p}, t)=f(\mathbf{r}, \mathbf{p}, t)-f_{0}(p),
$$

where $f_{0}(p)$ is the static global-equilibrium distribution 
function

$$
f_{0}(p)=\frac{n}{(2 m \pi T)^{3 / 2}} \exp \left(-\frac{p^{2}}{2 m T}\right) .
$$

The function $f_{0}(p)$ (3) is taken in the Maxwell form with constant values of $T$ and $n$ and zero value of the collective velocity, $\mathbf{u}=0$. The damping plane wave (DPW) solutions are assumed to be a good approximation to the dynamical variations $\delta f$ at finite frequencies $\omega$ within the FC regime (large-enough collision frequency $\nu$ ). These dynamical deviations allow us to take into account analytically the attractive VDW interactions through the self-consistent Vlasov mean field in the BVKE. As a result, we obtain the shear viscosity dependence on the particle number density at the leading order in a small parameter $\omega / \tau$. The overdamped (see, e.g., Refs. $[18,28]$ ) attenuation of the DPW will be considered. Our approach is based on the methods applied earlier for calculations of the viscosity of the Fermi liquids $[13,14,16,17,28]$. In the present paper, the shear viscosity in the first order over small parameter $\omega / \nu$ is calculated analytically for the nuclear matter considered as a gas of interacting nucleons with the VDW equation of state.

The paper is organized as follows. In Sec. II we remind the basic properties of thermodynamically equilibrated systems with the VDW equation of state. In Sec. III we outlook the kinetic approach based on the BVKE and give general definitions of the shear viscosity coefficient. In Sec. IV, the solution to the BVKE and its perturbation expansion is presented in terms of the plane waves accounting for a strong attenuation owing to the particle collisions. Finally, this section is devoted to the main results for the VDW viscosity. The obtained results are discussed in Sec. V and summarized in Sec. VI. Some details of our calculations can be found in Appendixes A-C.

\section{VDW EQUATION OF STATE}

The VDW equation of state presents the system pressure $P$ in terms of the particle number density $n$ and temperature $T$ as [29],

$$
P(T, n)=\frac{n T}{1-b n}-a n^{2},
$$

where $a>0$ and $b>0$ are the VDW parameters that describe attractive and repulsive interactions, respectively. The first term on the right-hand side of Eq. (4) contains the excluded volume correction $\left(b=2 \pi d^{3} / 3\right.$, with $d$ being the particle hard-core diameter), while the second term comes from the mean-field description of attractive interactions.

The entropy density $s$ and energy density $\varepsilon$ for the VDW system are calculated as [29]

$$
\begin{aligned}
& s(T, n)=\frac{5}{2} n+n \ln \left[\frac{(1-b n)}{n} g\left(\frac{m T}{2 \pi}\right)^{3 / 2}\right], \\
& \varepsilon(T, n)=n\left[\frac{3}{2} T-a n\right] .
\end{aligned}
$$

In Eq. (5) $m$ is the particle mass and $g$ is the degeneracy factor ( $g=4$ for nucleons; two spin and two isospin states). Note that the VDW entropy density (5) is independent of the attractive mean-field interaction parameter $a$, whereas the energy density (6) does not depend on the particle repulsion constant $b$.

The VDW equation of state contains the first-order liquid-gas phase transition with a critical point [29]:

$$
T_{c}=\frac{8 a}{27 b}, \quad n_{c}=\frac{1}{3 b}, \quad P_{c}=\frac{a}{27 b^{2}} .
$$

To study the phase coexistence region which exists below the critical temperature, $T<T_{c}$, the VDW isotherms should be corrected by the well-known Maxwell construction of equal areas.

The VDW equation of state was recently applied to a description of nuclear matter in Ref. [30]. In the present study we fix the VDW parameters for the system of interacting nucleons as $d=1 \mathrm{fm}$, i.e., $b \cong 2.1 \mathrm{fm}^{3}$, and $a=100 \mathrm{MeV} \mathrm{fm}^{3}$. This gives $n_{c} \cong n_{0}=0.16 \mathrm{fm}^{-3}$ and $T_{c} \cong 14 \mathrm{MeV}\left(n_{0}=0.16 \mathrm{fm}^{-3}\right.$ corresponds to the nucleon number density of the normal nuclear matter at zero temperature). In what follows we restrict our analysis of the kinetic properties of the VDW system of nucleons to $T>T_{c}$. In this region of the phase diagram the VDW equation of state describes a homogeneous onephase system, and all criteria of the thermodynamical stability are satisfied. We do not consider too large temperatures by taking $T \lesssim 40 \mathrm{MeV}$. This allows us to neglect a production of new particles (pions and baryonic resonances) in the system of interacting nucleons. In addition, this restriction guarantees a good accuracy of the nonrelativistic approximation adopted in the present study. Note also that at $T \rightarrow 0$ the quantum statistics effects neglected in the present study should be taken into account (see Ref. [30]).

\section{KINETIC APPROACH}

For calculations of the shear viscosity, we start with the BVKE linearized near the static distribution function (3) for the dynamical variations of the distribution function $\delta f(\mathbf{r}, \mathbf{p}, t)(2)$ :

$$
\frac{\partial \delta f}{\partial t}+\frac{\mathbf{p}}{m} \frac{\partial \delta f}{\partial \mathbf{r}}-\frac{\partial f_{0}}{\partial \mathbf{p}} \frac{\partial \delta U}{\partial \mathbf{r}}=\delta S t .
$$

The dynamical part of the attractive potential $\delta U$ from the VDW forces is defined self-consistently as

$$
\delta U(\mathbf{r}, t)=-a \int \mathrm{d} \mathbf{p} \delta f(\mathbf{r}, \mathbf{p}, t) .
$$

In Eq. (8), the collision term $\delta S t$ is taken in the standard Boltzmann form $[5,8]$,

$$
\delta S t=\frac{2 \pi}{m} \int \mathrm{d} \mathbf{p}_{1}\left|\mathbf{p}_{1}-\mathbf{p}\right| \int \beta \mathrm{d} \beta \delta Q,
$$

where

$$
\begin{aligned}
\delta Q & \approx f_{0}\left(p^{\prime}\right) \delta f\left(\mathbf{r}, \mathbf{p}_{1}^{\prime}, t\right)+f_{0}\left(p_{1}^{\prime}\right) \delta f\left(\mathbf{r}, \mathbf{p}^{\prime}, t\right) \\
& -f_{0}(p) \delta f\left(\mathbf{r}, \mathbf{p}_{1}, t\right)-f_{0}\left(p_{1}\right) \delta f(\mathbf{r}, \mathbf{p}, t)
\end{aligned}
$$




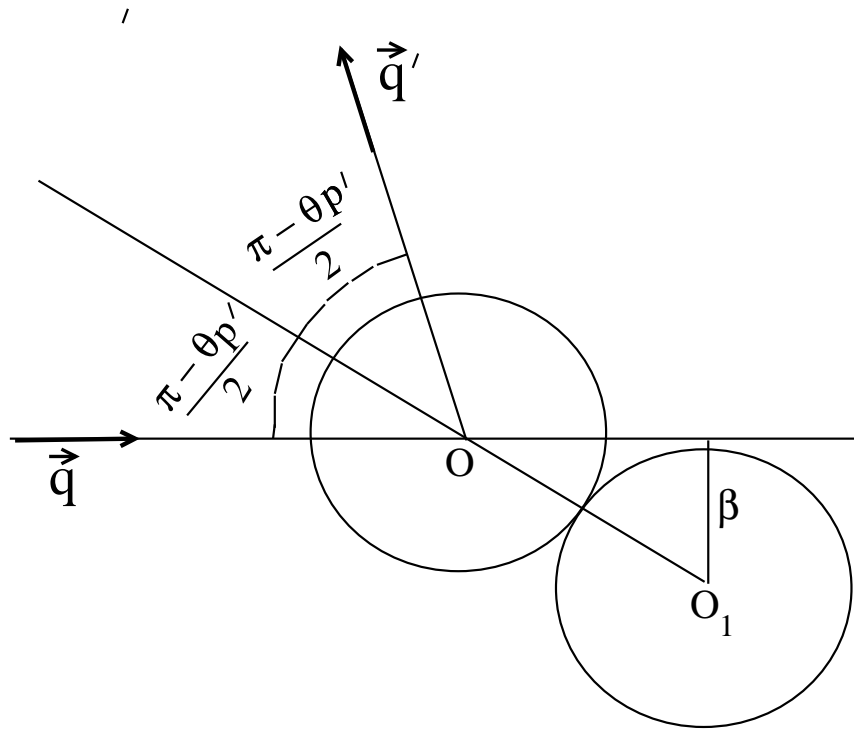

FIG. 1. The geometry of the collision of two hard spheres in the center mass system; $\mathbf{q}=\mathbf{p}_{1}-\mathbf{p}, \mathbf{q}^{\prime}=\mathbf{p}_{1}^{\prime}-\mathbf{p}^{\prime}, O O_{1}=d$, $d$ is the sphere particle diameter, $\beta$ is the impact parameter; $\theta_{p^{\prime}}$ is the scattering angle.

is the variation of $f\left(\mathbf{r}, \mathbf{p}^{\prime}, t\right) f\left(\mathbf{r}, \mathbf{p}_{1}^{\prime}, t\right) \quad-$ $f(\mathbf{r}, \mathbf{p}, t) f\left(\mathbf{r}, \mathbf{p}_{1}, t\right)$ over $\delta f$ and $\beta$ the impact parameter for two-body collisions. Figure 1 shows the collision geometry for two hard-core sphere scattering in the center-of-mass coordinates. The relationship between the impact parameter, $\beta$, and the cross section, $\sigma=\pi d^{2}$, where $d$ is the diameter of the particle, is given in Appendix A for calculations of the integral term (10).

The shear viscosity $\eta$ can be defined through the dynamical components of the momentum flux tensor $\Pi_{\mu \nu}(\mathbf{r}, t)$,

$$
\delta \Pi_{\mu \nu}=-\delta \sigma_{\mu \nu}+\delta \mathcal{P} \delta_{\mu \nu}+\delta P \delta_{\mu \nu},
$$

where $\delta \sigma_{\mu \nu}$ is a traceless stress tensor. Other terms are diagonal kinetic and interaction pressures. The stress tensor $\delta \sigma_{\mu \nu}$ can be determined through the second $\mathbf{p}$ moment of the distribution function linearized over $\delta f$ as

$$
\delta \sigma_{\mu \nu}=-\int \frac{\mathrm{d} \mathbf{p}}{m} p_{\mu} p_{\nu} \delta f(\mathbf{r}, \mathbf{p}, t)+\delta \mathcal{P} \delta_{\mu \nu} .
$$

In Eq. (12), the quantities $\delta \mathcal{P}$ and $\delta P$ are calculated as

$$
\begin{aligned}
& \delta \mathcal{P}=\frac{1}{3 m} \int p^{2} \mathrm{~d} \mathbf{p} \delta f(\mathbf{r}, \mathbf{p}, t), \\
& \delta P=-2 a n \int \mathrm{d} \mathbf{p} \delta f(\mathbf{r}, \mathbf{p}, t) .
\end{aligned}
$$

The shear viscosity $\eta$ is defined as a coefficient in the relationship between the dynamical component of the stress tensor $\delta \sigma_{\mu \nu}$ [Eq. (12)] and the traceless tensor $\mathcal{U}_{\mu \nu}$ of the coordinate derivatives of the velocity field $\mathbf{u}(\mathbf{r}, t)$ $[10,14,15,17,31]$,

$$
\delta \sigma_{\mu \nu}(\mathbf{r}, t)=\eta \mathcal{U}_{\mu \nu}(\mathbf{r}, t),
$$

where

$$
\mathcal{U}_{\mu \nu}=\left(\frac{\partial u_{\mu}}{\partial r_{\nu}}+\frac{\partial u_{\nu}}{\partial r_{\mu}}-\frac{2}{3} \nabla \mathbf{u} \delta_{\mu \nu}\right)
$$

The velocity field $\mathbf{u}$ is defined through the first $\mathbf{p}$ moment of $\delta f(\mathbf{r}, \mathbf{p}, t)$,

$$
\mathbf{u}=\frac{1}{n} \int \mathrm{d} \mathbf{p} \frac{\mathbf{p}}{m} \delta f(\mathbf{r}, \mathbf{p}, t) .
$$

Note that our method can also be presented within the linear response-function theory [15, 28, 32] (cf. the Kubo formulas for the diffusion, thermal conductivity, and viscosity; see also the recent article [33]).

\section{DISPERSION RELATION AND VISCOSITY}

We suggest to calculate the shear viscosity $\eta$ by directly solving the BVKE (8) in terms of the plane-wave representation for the dynamical distribution-function variations $\delta f(\mathbf{r}, \mathbf{p}, t)[\mathrm{Eq} .(2)]$ in the following rather general form $[14,17,28]$,

$$
\delta f(\mathbf{r}, \mathbf{p}, t)=f_{0}(p) \varphi(\hat{p}) \exp (-i \omega t+i \mathbf{k r}),
$$

where $\omega$ and $\mathbf{k}$ are a frequency and a wave vector of the DPW, respectively. As unknown yet, amplitudes $\varphi(\hat{p})$ are functions of the momentum angle variable $\hat{p}=\mathbf{p} / p$. It is naturally to find solutions of the BVKE as proportional to the static distribution function, $f_{0}(p)$, specifying the dependence of $\delta f$ on the modulus of momentum $p$ because the derivative of $f_{0}(p)(3)$ over momentum in Eq. (8) and the variations of the collision integral (10) are proportional to $f_{0}(p)$. Then, one can reduce the problem for solving the BVKE (8) to a function of angles $\varphi(\hat{p})$ which, however, depends on the uknown frequency $\omega$. [We shall leave out the argument $\omega$ in $\varphi(\hat{p})$ for simplicity of the notations.] Note that any physical quantity, in particular the viscosity coefficient, is independent of the direction of the unit wave vector $\hat{k}=\mathbf{k} / k$ of the DPW spreading in infinite nuclear matter. Therefore, it is convenient to use the spherical phase-space coordinate system with the polar axis directed to this vector $\hat{k}$. The solution for the plane-wave distribution function $\delta f(\mathbf{r}, \mathbf{p}, t)[$ Eq. (19)] or more precisely $\varphi(\hat{p})$, and the frequency $\omega$ depends only on the wave vector length $k$. For convenience, one may write the frequency $\omega$ through the wave number $k$ and the dimensionless sound velocity $c$,

$$
\omega=k v=k v_{T} c,
$$

where $v=v_{T} c$ is the DPW speed, and $c$ its dimensionless value given in units of the most probable thermal velocity $v_{T}$ of particles at a given temperature $T, v_{T}=\sqrt{2 T / m}$.

The viscosity $\eta$ is related to an attenuation of the DPW (19) measured by the collision term $\delta S t$ (10). Following Refs. [5, 8, 9, 14], one applies the perturbation expansion of the dynamical distribution-function variations $\delta f$ through their amplitudes $\varphi(\hat{p})$,

$$
\varphi(\hat{p})=\varphi^{(0)}(\hat{p})+\epsilon \varphi^{(1)}(\hat{p})+\epsilon^{2} \varphi^{(2)}(\hat{p})+\ldots,
$$


and similarly, in addition to Ref. [5], for the frequency $\omega$

$$
\omega=\omega^{(0)}+\epsilon \omega^{(1)}+\epsilon^{2} \omega^{(2)}+\ldots,
$$

in a small parameter,

$$
\epsilon=\omega / \nu=\omega \tau \text {. }
$$

Here, $\tau$ is the relaxation time ${ }^{1}$ defined by the collision term through the time-dependent rate $\nu$ (collision frequency) of the damping of distribution function $\delta f$,

$$
\tau=1 / \nu .
$$

Expansions (21) and (22) are defined within the standard perturbation method $[8,14,28,34]$ for the eigenfunction, $\varphi(\hat{p})$, and eigenvalue, $\omega$, problem. In these perturbation expansions, the coefficients $\varphi^{(n)}(\hat{p})$ and $\omega^{(n)}$ are assumed to be independent of $\epsilon$. By using the BVKE with this perturbation method, they can be found at each order of $\epsilon$. Note that $\omega$ in the definition of the small parameter $\epsilon$ (23) is determined consistently at any given order of the perturbation expansions (21) and (22); see Appendix B for details. A smallness of $\epsilon$ can be achieved by increasing the collision frequency $\nu$ for a given $\omega$ (Appendix A). Substituting the plane-wave representation (19) for the distribution function $\delta f$ into the BVKE (8), for convenience, one can also expand $\varphi(\hat{p})$ in series over the spherical harmonics $Y_{\ell 0}(\hat{p})$,

$$
\varphi(\hat{p})=\sum_{\ell=0}^{\infty} \varphi_{\ell} Y_{\ell 0}(\hat{p}), \quad \hat{p}=\frac{\mathbf{p} \cdot \mathbf{k}}{p k} .
$$

This reduces the integro-differential BVKE to much more simple linear algebraic equations (B.1) for the partial multipole amplitudes $\varphi_{\ell}$ at each order in $\epsilon$ [Eq. (23) and Appendix B].

As shown in Appendixes $\mathrm{A}$ and $\mathrm{B}$, in the $\mathrm{FC}$ regime, $|\epsilon| \ll 1$ [Eq. (23)], one can truncate the multipole expansion (25) over $\ell$ at $\ell=2$ because of a good convergence in the small parameter $\epsilon$. At this leading approximation to viscosity calculations, for the collision term $\delta S t$ [Eq. (10)], one obtains (Appendix A) the simple expression

$$
\delta S t=-\nu \delta f_{2}(\mathbf{r}, \mathbf{p}, t),
$$

where

$$
\nu \approx \frac{3 n v_{T} \sigma}{2}, \quad \sigma=\pi d^{2},
$$

$\sigma$ is the cross section for a two elastic hard-core sphere scattering, as introduced above,

$$
\delta f_{2}(\mathbf{r}, \mathbf{p}, t)=f_{0}(p) \varphi_{2} Y_{20}(\hat{p}) \exp (-i \omega t+i \mathbf{k r}) .
$$

As shown in Appendix A, within the accuracy about $6 \%$, this value agrees with its mean effective quantity $\nu_{a v}$

\footnotetext{
${ }^{1}$ We do no not use the standard $\tau$ approximation and introduce the relaxation time $\tau$ for sake of the convenience in comparison with other approaches.
}

(A.18), evaluated through the momentum average of the collision term, $\langle\delta S t\rangle_{\mathrm{av}}$, over particle momenta $p$ with the help of the Maxwell distribution $f_{0}(p)(3)$. Multiplying then the BVKE (8), with the quadrupole collisional term (26), by the spherical function $Y_{L 0}(\hat{p})(L=0,1,2, \ldots)$, one can integrate the BVKE term by term over angles $(\hat{p})$ of the momentum p. Thus, one obtains the linear homogeneous equations (B.1) with respect to coefficients $\varphi_{\ell}$ of the expansion (25) in the plane-wave amplitudes $\varphi(\hat{p})$ at any order in $\epsilon$ in Eqs. (21) and (22). This system has nontrivial solutions in the quadrupole approximation $\ell \leq 2$, valid at the leading (linear in $\epsilon$ ) approximation in expansions (21) and (22). They obey the cubic dispersion equation for $c=\omega /\left(k v_{T}\right)$ (expansion of $c$ is similar to Eq. (22); see also Appendix B),

$$
\begin{gathered}
\operatorname{det} \mathcal{A}_{2} \equiv c^{3}+i \gamma c^{2}-c\left[\frac{4}{15}+\frac{1}{3}(1-\mathcal{F})\right] \\
-\frac{i}{3}(1-\mathcal{F}) \gamma=0
\end{gathered}
$$

where $\mathcal{F}$ is the dimensionless VDW interaction parameter,

$$
\mathcal{F}=a n / T
$$

The truncated (at $\ell=2) 3 \times 3$ matrix $\mathcal{A}_{L \ell}^{(2)}(c)$ is given by Eq. (B.4). For convenience, we introduced also the dimensionless collisional rate (27):

$$
\gamma=\frac{\nu}{k v_{T}}=\frac{\nu c}{\omega}=\frac{c}{\omega \tau} .
$$

The FC perturbation parameter $\epsilon$ [Eq. (23)] can be expressed in terms of the $\gamma$ and $c$ as

$$
\epsilon=c / \gamma, \quad|c / \gamma| \ll 1 .
$$

The cubic dispersion equation (29) has still two limit solutions with respect to the complex velocity, $c=$ $c_{r}+i c_{i}$ for real $k$ (or equivalently, a complex wave number $k=k_{r}+i k_{i}$ for a real velocity $c$, both related by the same $\omega=k c v_{T}=\omega_{r}+i \omega_{i}$, where low subscripts denote the real and imaginary parts). One of them can be called as the underdamped (weakly damped) first sound mode for which the imaginary part of $c, c_{i}$, is much smaller than the real one $c_{r},\left|c_{i} / c_{r}\right| \ll 1$, while in the opposite case $\left|c_{i} / c_{r}\right| \gg 1$, one has the overdamped motion. In the first underdamped sound case $\left(\left|c_{i} / c_{r}\right| \ll 1\right)$, the collision term can be considered as small with respect to the left-hand side (LHS) of the BVKE, $|\gamma / c| \sim 1 /|\omega \tau| \ll 1$, that is, the rare collision $(\mathrm{RC})$ regime. For the overdamped motion (FC case) the collision term is dominant. In our DPW derivations below one can use also the frequency expansion (22) over the same small parameter $|c / \gamma| \sim|\omega \tau| \ll 1$ [ Eqs. (31) and (32)]. In the present study we consider the overdamped motion, while the underdamped case will be studied in separate publications.

Expanding the LHS of the truncated (quadrupole) dispersion equation (29) for $c$ in powers of $\epsilon$ [see Eqs. (23) and (32)] in the FC perturbation expansions (21) and (22), one can divide all of its terms by $\gamma^{3}$. Then, one can neglect the relatively small cubic $\left[(c / \gamma)^{3} \sim \epsilon^{3}\right]$ and quadratic $\left(\sim \epsilon^{2}\right)$ terms as compared to the last two linear (in $\epsilon$ ) ones depending explicitly on the interaction 


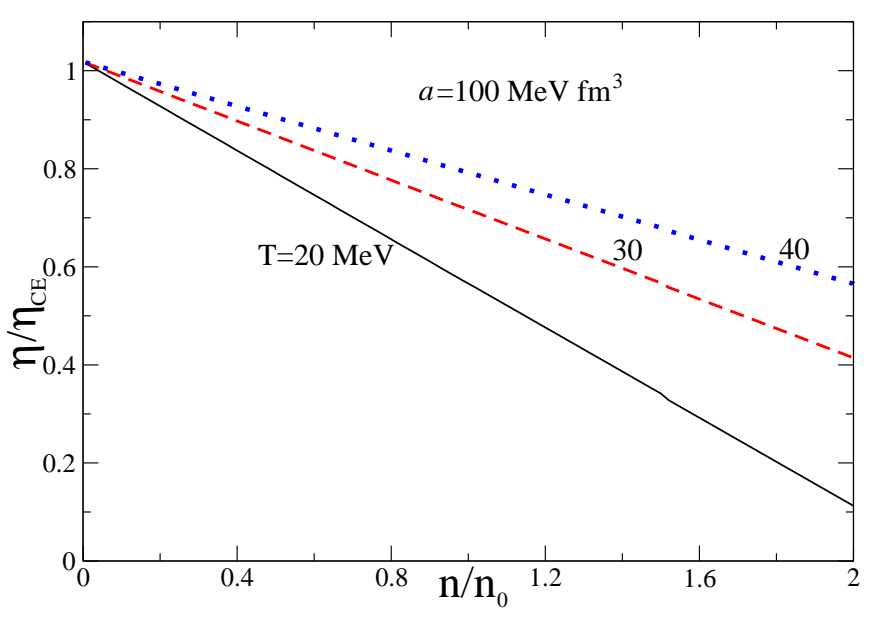

FIG. 2. Shear viscosity $\eta$ [Eq. (36)] in the frequent-collision regime in units of the $\mathrm{CE}$ value $\eta_{\mathrm{CE}}[\mathrm{Eq}$. (1)] versus particlenumber density $n$ in units of the normal density $n_{0}=0.16$ $\mathrm{fm}^{-3}$ of nuclear matter; $m \cong 938 \mathrm{MeV} ; d=1 \mathrm{fm}, a=$ $100 \mathrm{MeV} \mathrm{fm}^{3}$.

parameter $\mathcal{F}$ [Eq. (30)]. At this leading order, one results in the explicit quadrupole solution for the velocity $c$ [Eq. (B.7)],

$$
c=i c_{i}=-\frac{5 i}{9} \frac{1-\mathcal{F}}{1-5 \mathcal{F} / 9} \gamma .
$$

To get small corrections of the real sound velocity $c_{r}$, one has to take into account the quadratic and cubic in $c$ terms of the dispersion equation (29). Note that formally, one can consider the real DPW velocity $c$ but the complex wave number $k$ within the same complex frequency $\omega$, which are both almost pure imaginary ones. The latter describes the sound attenuation as the exponential decrease of the DPW amplitude, $\delta f \propto \exp (-t / \mathcal{T})[\mathrm{Eq} .(19)]$ with the damping time $\mathcal{T}$,

$$
\mathcal{T} \approx \frac{6}{5 \pi} \frac{1-5 \mathcal{F} / 9}{(1-\mathcal{F}) n v_{T} d^{2}} .
$$

This time was obtained as the imaginary part of the complex frequency, $\omega=-i / \mathcal{T}$, through Eq. (33), formally introduced above (finally, all physical quantities will be determined by taking their real parts). Note also that the relaxation time $\tau$ [Eqs. (24) and (27)],

$$
\tau=\frac{1}{\nu} \approx \frac{2}{3 n v_{T} \pi d^{2}},
$$

differs from the damping time, $\mathcal{T}$ [Eq. (34)]. In particular, this time $\mathcal{T}$, being of the order of $\tau$, depends on the interaction constant $\mathcal{F}$. Note that the $\mathrm{FC}$ condition (32) can be satisfied for the interaction parameter $\mathcal{F}$ of the order of one. However, as shown below, one finds a reasonable result even in the limit $\mathcal{F} \rightarrow 0$.

Using the DPW solutions (19) for $\delta f$ of the BVKE (8), and Eqs. (C.3) for $\mathcal{U}_{z z}$ and (C.5) for $\sigma_{z z}$, for the definition of the shear viscosity $\eta[\mathrm{Eq} .(16)]$, one finds the FC expansions [(21) and (22)] of $\eta$ in powers of small $\epsilon$ [see Eq. (23), and Appendixes C and B]. As shown in Appendix $\mathrm{C}$, the leading term of this $\mathrm{FC}$ shear viscosity $\eta$ at first order in $\epsilon$ is approximately a constant, independent of $\omega$ (or $k$ ), and proportional to $1 / \nu$, i.e., to the relaxation time $\tau$ [Eq. (35)]. Finally, up to relatively high (second) order terms in the small parameter $\epsilon$ we arrive at

$$
\begin{aligned}
\eta & =\frac{9}{20 \sqrt{2 \pi}}\left(1-\frac{5}{9} \frac{a n}{T}\right) \frac{\sqrt{m T}}{d^{2}}=\frac{36}{25 \sqrt{2}}\left(1-\frac{5}{9} \frac{a n}{T}\right) \eta_{\mathrm{CE}} \\
& =1.018\left(1-\frac{5}{9} \frac{a n}{T}\right) \eta_{\mathrm{CE}} \cdot
\end{aligned}
$$

In these derivations we used, at the leading first order in $\epsilon$, the quadrupole multipolarity truncation of rapidly converged series (25); see Eqs. (C.8) for the amplitudes $\varphi_{\ell}$ and (33) for the sound velocity $c(\ell \leq 2)$ within the dispersion equation (29). As seen from Eq. (36), within the present accuracy, the shear viscosity $\eta$ differs in 2 $\%$ from the $\mathrm{CE}$ result $\eta_{\mathrm{CE}}[\mathrm{Eq}$. (1)] at zero attractive mean field, $a \rightarrow 0$. Note that a more exact $\mathrm{CE}$ result is $\eta=1.016 \eta_{\mathrm{CE}}$ (see Ref. [5], Chap. 12.1).

Formula (36) for the shear viscosity can be presented in a more traditional way through the relaxation time $\tau$ [Eq. (35)],

$$
\eta=\frac{27 \sqrt{\pi}}{80}\left(1-\frac{5}{9} \mathcal{F}\right) m n v_{T}^{2} \tau .
$$

This relationship, $\eta \propto \tau$, is typical for the $\mathrm{FC}$ regime, in contrast to the rare collision one, $\eta \propto 1 / \tau$, which should be expected for the perturbation expansion at leading order in the opposite small parameter $1 / \epsilon$; see Refs. [14, 16, 17, 28]. Note that the perturbation method for the eigenfunctions $\varphi(\hat{p})$ (or $\varphi_{\ell}$, Eq. (21) as in Ref. [5])), and in addition, eigenvalues $\omega$ allows us to obtain in a regular way high-order corrections in $\epsilon$. In this way, one has to go beyond the quadrupole multipolarity $(\ell \leq$ 2 ) approximation taking into account, consistently at a given $\epsilon$, higher order terms, $\ell>2$, in expansion (25) for $\varphi(\hat{p})$.

\section{DISCUSSION OF THE RESULTS}

Equation (36) for $\eta$ has the same classical hydrodynamical dependence on the temperature $T$ and diameter $d, \eta \propto \sqrt{m T} / d^{2}$ [cf. with Eq. (1)], because of using the FC approximation as in both the molecular kinetic theory [10] and the CE approach [5]. In this approximation for the overdamped case (Appendix B) the dominating contribution into the viscosity yields from the collision term which mainly determines both the classical hydrodynamical solutions (Ref. [5]) and our DPW ones [Eq. (19)] for the distribution function to the BVKE. Therefore, as expected, in the limit $a \rightarrow 0(\mathcal{F} \ll 1)$, one finds the number constant [in front of $\sqrt{m T} / d^{2}$; see Fig. 2 and Eq. (36)] that approximately coincides within the accuracy of $2 \%$ with the CE result (1). The difference between the hydrodynamical (1) and overdamped DPW (36) viscosities in the zero interaction constant 


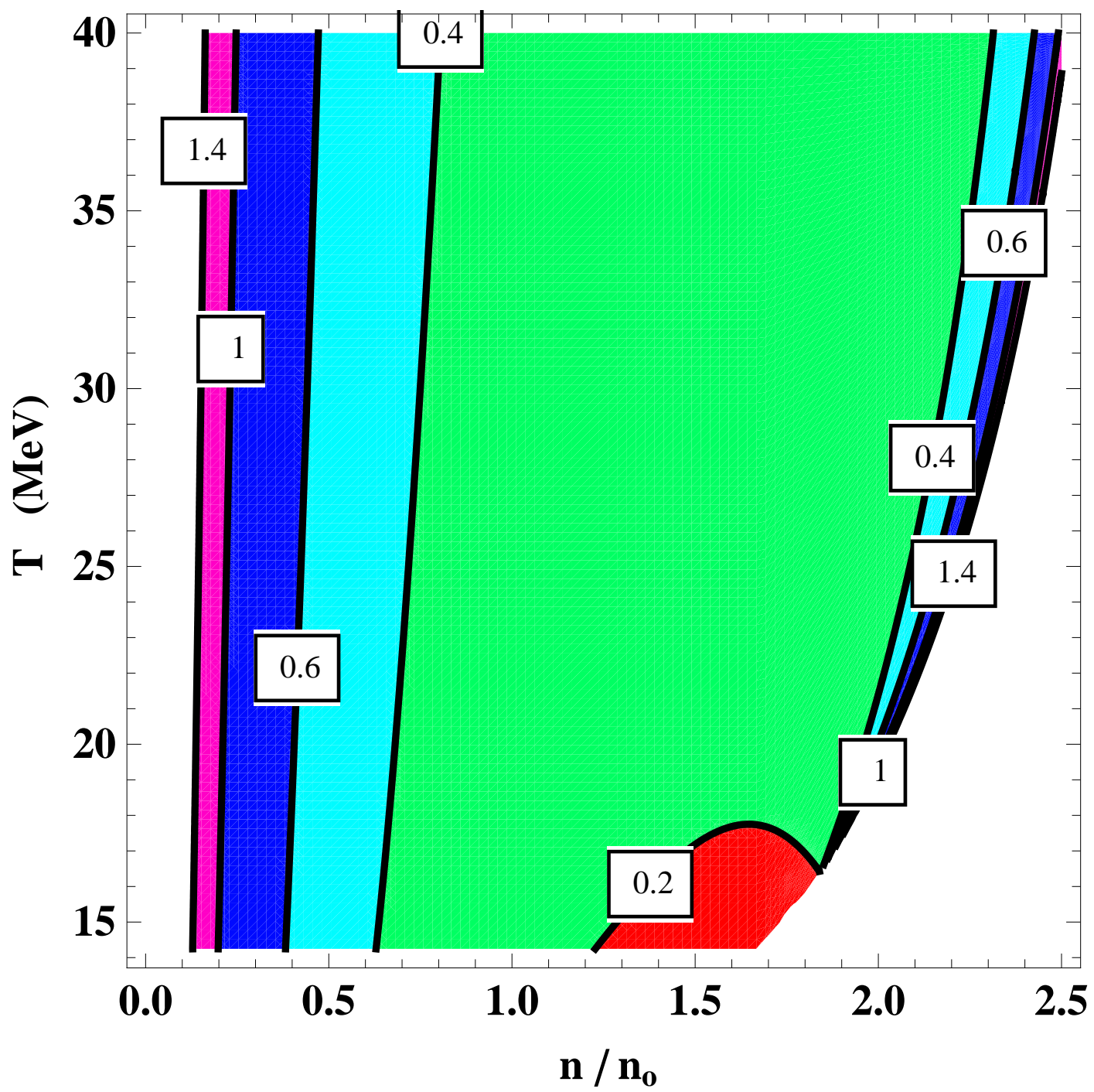

FIG. 3. Contour plot for the ratio $\eta / s$ of the DPW viscosity $\eta[$ Eq. (36)] to the entropy density $s(5)$ in the $n-T$ plane at $g=4$ and the same $m, d$, and $a$ parameters as in Fig. 2.

limit should be, indeed, small as compared to the leading collisional term.

Figure 2 shows the shear viscosity $\eta$ [Eq. (36)] for a few temperatures above the critical value $T_{c}$ [Eq. (7)]. From Fig. 2, one can clearly see that the shear viscosity $\eta$ differs significantly from the classical hydrodynamical formula (1) by the particle density dependence. It appears through the VDW parameter $\mathcal{F}$ [Eq. (30)], owing to accounting for dynamical variations of the mean-field interaction (9) in our derivations. As displayed in this figure, the significant effects originate by the Vlasov selfconsistent attractive-interaction terms of the BVKE. In our approach this is achieved by solving the BVKE (8) in terms of the DPW nonlocal-equilibrium distribution function $\delta f$ [see Eq. (19)] and using, therefore, the perturbation expansion (22) for the frequency $\omega$ as a solution of the dispersion equation, in addition to Eq. (21). This is in contrast to the CE approach based on the dynamical local-equilibrium distribution-function variations and hydrodynamical equations, used on the lefthand side of the BVKE. The interaction term of the Boltzmann kinetic equation containing $\delta U$ [Eqs. (9)] is neglected in the CE method [5] as compared to the integral collision term of the BVKE at the leading first order in $\epsilon$. Therefore, there is no particle density corrections to the shear viscosity in the CE approach at this order. To obtain these corrections, we found another alternative DPW solution (19) through the self-consistent interaction term of the BVKE. Note also that with increasing attractive interaction parameter $a(a>0)$, one finds a linearly decreasing viscosity $\eta$ through the dimensionless parameter $\mathcal{F}$.

Figures 3 and 4 show the ratio, $\eta / s$, of the viscosity $\eta$ to the entropy density $s$ [Eq. (5)] given by Eqs. (36) and (1), respectively, in the $n-T$ plane for temperatures $T$ above the critical value $T_{c}$ [Eq. (7)]. As seen from comparison of the two overdamped viscosities in units of the entropy density in these figures, the ratio $\eta / s$ takes form of a minimum with values $\eta / s \lesssim 1$ at densities $(0.5-2) n_{0}$, somewhere in a vicinity of the critical point $\left(T_{c}, n_{c}\right)$. This minimum is significantly smaller and moves to smaller temperatures in our DPW calculations (Fig. 3) as compared to the CE ones (Fig. 4) though they are both smaller than 1 . Note also a weak sensitiv- 


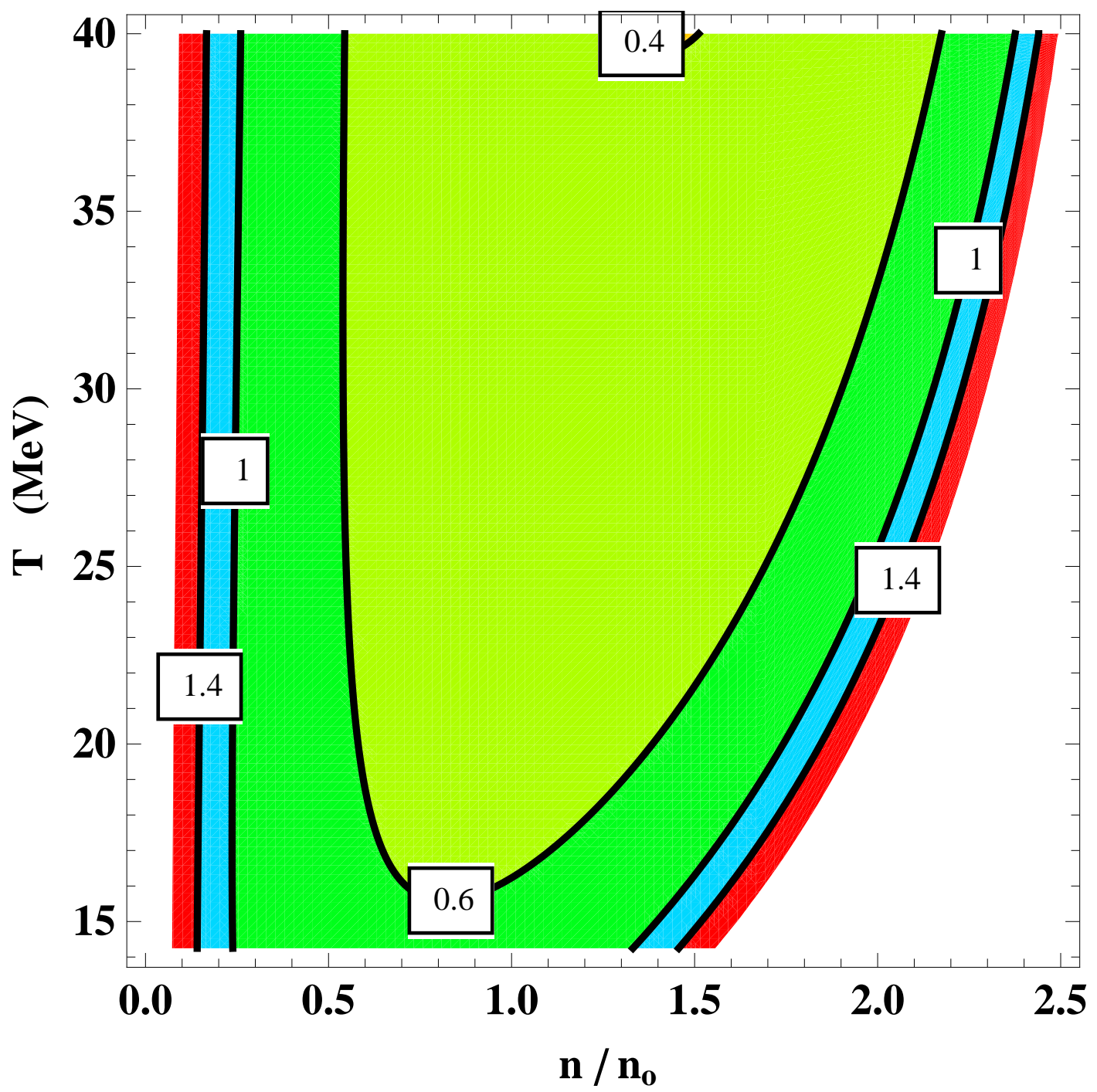

FIG. 4. Same as in Fig. 3 but for the ratio of the hydrodynamic CE shear viscosity [Eq. (1)] to the entropy density [Eq. (5)].

ity of these properties depending of the size of the hard spheres $d$ around $d=1 \mathrm{fm}$ for its deflections in about $20 \%$. However, $\eta / s \gg 1$ both at small $\left(n \ll n_{0}\right)$ and large $\left(n \gtrsim 2 n_{0}\right)$ particle density, which makes the ideal hydrodynamic approach inapplicable for these densities. We should emphasize that the BVKE can be applied for enough dilute system of particles where the mean free path is large as compared to particles' interaction region (in our example, of the order of the size of particles $d$ ). This gas condition should be satisfied for all desired densities.

\section{CONCLUSIONS}

The shear viscosity of a nucleon gas is derived by solving the BVKE for the FC regime with taking into account the van der Waals interaction parameters for both the hard-elastic sphere scattering and attractive meanfield interaction. The viscosity $\eta$ depends on the particle density $n$ through the dynamical mean-field forces measured by the VDW parameter, an/T, which is positive for the attractive long-distance mean-field interaction.
Therefore, the viscosity $\eta$ decreases with the interaction constant $a>0$ through the VDW parameter $\mathcal{F}$. The ratio of the $\mathrm{FC}$ viscosity to the entropy density, $\eta / s$, as function of the particle density $n$ and temperature $T$ is found to have a minimum which is essentially smaller than one. The viscosity is significantly smaller at this minimum which moves to smaller temperatures toward the critical temperature owing to the long-distance interaction, as compared to the classical hydrodynamical CE result. Our DPW viscosity calculations have the same overdamped behavior (strong attenuation) such that the collisional term is dominating above all of other parts of the BVKE. Note that the viscosity coefficient can be consider as a response (Ref. [15, 28, 32, 35]) of the stress tensor $\sigma_{\mu \nu}$ for the shear pressure on the velocity derivative tensor $\mathcal{U}_{\mu \nu}$; see, e.g., Eq. (16). See also the Green'sKubo formula for the shear viscosity, as for the conductivity coefficient [15, 33].

Our results might be interesting for the kinetic and hydrodynamic studies of nucleus-nucleus collisions at laboratory energies of a few hundreds $\mathrm{MeV}$ per nucleon. The ideal hydrodynamics can be a fairly good approximation for a system of the interacting nucleons in the region 
of $n$ and $T$ that corresponds at least to $\eta / s \ll 1$. However, the classical hydrodynamical approach for both the dilute nucleon gas with $n \ll n_{0}$ and the nuclear-dense matter with $n \gtrsim 2 n_{0}$ seems to be rather questionable to use. As a different perturbation theory has to be used in expansions over small $\omega \tau$ for the FC and small $1 /(\omega \tau)$ in the $\mathrm{RC}$ regime, we should expect very different dependencies of the viscosity (and other transport coefficients) on the particle density $n$ in these two opposite limits. For instance, the RC regime is important to study a weak absorption of the DPW in the gas system with small far-acting interactions, especially for ultrasonic absorption $[36,37]$. Therefore, in the case when the contributions of collisions into the BVKE dynamics are changed from the dominant (small $\epsilon$ ) to almost collisionless process (small $1 / \epsilon$ ) with increasing DPW frequency for a given collision frequency $\nu$, a transition from the $\mathrm{FC}$ to $\mathrm{RC}$ regimes should be accounted beyond the classical hydrodynamical approach. This can be realized for small $n / n_{0}$ and large $\eta / s$, in the corresponding $n-T$ regions of the phase diagram for analysis of the nucleus-nucleus collisions. Our approach can be applied to calculations of the thermal conductivity and diffusion coefficients in nuclear physics, as well as those and viscosity in nuclear astrophysics, and to study different phenomena in the electron-ion plasma.

\section{ACKNOWLEDGMENTS}

We thank D.V. Anchishkin, S.N. Reznik and A.I. Sanzhur for fruitful discussions. The work of M.I.G. was supported by the Program of Fundamental Research of the Department of Physics and Astronomy of National Academy of Sciences of Ukraine. One of us (A.G.M.) is very grateful for the financial support of the Program of Fundamental Research to develop further cooperation with CERN and JINR "Nuclear matter in extreme conditions" by the Department of Nuclear Physics and Energy of National Academy of Sciences of Ukraine, Grant No. CO-2-14/2016, for nice hospitality during his working visit to the Nagoya Institute of Technology, and also for financial support from the Japanese Society of Promotion of Sciences, Grant No. S-14130.

\section{Appendix A: COLLISION TERM CALCULATIONS}

We shall neglect approximately an influence of the effective potential $\delta U[n(\mathbf{r}, t)]$ (9) of the long-range particle interaction during a two-particle collision of hard-core sphere particles of the gas in the $\mathrm{FC}$ regime. Using the multipole expansion (25) of the amplitude factor $\varphi(\hat{p})$, one can simplify the linearized collisional term, $\delta S t$ [Eqs. (10) and (19)], in the BVKE (8),

$$
\begin{aligned}
& \quad \delta S t=\frac{d^{2}}{4 m} \sum_{\ell} \chi_{\ell} \int \mathrm{d} \mathbf{p}_{1}\left|\mathbf{p}_{1}-\mathbf{p}\right| \int \mathrm{d} \Omega_{p^{\prime}} \\
& \times\left\{f_{0}\left(p_{1}^{\prime}\right) f_{0}\left(p^{\prime}\right)\left[Y_{\ell 0}\left(\widehat{p}_{1}^{\prime}\right)+Y_{\ell 0}\left(\widehat{p}^{\prime}\right)\right]\right. \\
& \left.-f_{0}\left(p_{1}\right) f_{0}(p)\left[Y_{\ell 0}\left(\widehat{p}_{1}\right)+Y_{\ell 0}(\widehat{p})\right]\right\}
\end{aligned}
$$

where $f_{0}(p)$ is the static distribution function (3),

$$
\chi_{\ell}=\varphi_{\ell} \exp (-i \omega t+i \mathbf{k r}),
$$

and $\varphi_{\ell}$ is the $\ell$ coefficient in the expansion (25) for amplitudes $\varphi(\hat{p})$. One finds the relationship between the impact parameter $\beta$ in the center-of-mass coordinate system [see Fig. 1] and the scattering angle $\theta_{p^{\prime}}$ (and $\beta \mathrm{d} \beta$ to $\left.\mathrm{d} \Omega_{p^{\prime}}\right)$,

$$
\begin{aligned}
\beta & =\cos \left(\theta_{p^{\prime}} / 2\right) d, \quad \beta \mathrm{d} \beta= \\
& =\frac{d^{2}}{8 \pi} \mathrm{d} \Omega_{p^{\prime}}=\frac{d^{2}}{8 \pi} \sin \theta_{p^{\prime}} \mathrm{d} \theta_{p^{\prime}} \mathrm{d} \varphi_{p^{\prime}} .
\end{aligned}
$$

The Boltzmann collision term (A.1) is defined in such a way that its zero and first $\mathbf{p}$ moments have to be zero because of the particle-number conservation (related to the continuity equation), and momentum conservation,

$$
\mathbf{p}+\mathbf{p}_{1}=\mathbf{p}^{\prime}+\mathbf{p}_{1}^{\prime},
$$

(associated with the momentum continuity equation) during a two-body collision. We take also into account that the distribution function (3) is located within a small momentum interval $(2 m T)^{1 / 2}$. Within this range the momentum vectors are approximately changed only by their direction angles,

$$
\hat{p}+\hat{p}_{1} \approx \hat{p}^{\prime}+\hat{p}_{1}^{\prime},
$$

and one can use also the kinetic-energy conservation equation,

$$
p^{2}+p_{1}^{2}=p^{\prime 2}+p_{1}^{\prime 2} .
$$

Substituting Eq. (3) for the static distribution function, $f_{0}(p)$, and using the conservation equations (A.4)(A.6) in Eq. (A.1), one finds $f_{0}\left(p_{1}^{\prime}\right) f_{0}\left(p^{\prime}\right)=f_{0}\left(p_{1}\right) f_{0}(p)$. Therefore, from Eq. (A.1), one obtains

$$
\begin{gathered}
\delta S t=\frac{d^{2}}{4 m} f_{0}(p) \sum_{\ell} \chi_{\ell} \int \mathrm{d} \mathbf{p}_{1}\left|\mathbf{p}_{1}-\mathbf{p}\right| f_{0}\left(p_{1}\right) \\
\times \int \mathrm{d} \Omega_{p^{\prime}}\left[Y_{\ell 0}\left(\hat{p}_{1}^{\prime}\right)+Y_{\ell 0}\left(\hat{p}^{\prime}\right)-Y_{\ell 0}\left(\hat{p}_{1}\right)-Y_{\ell 0}(\hat{p})\right] .
\end{gathered}
$$

Thus, the collisional term (A.1) ensures all necessary (particle number, momentum, and energy) conservation laws. In particular, one can check that there is no $\ell=0$ and 1 terms in the sum over $\ell$ of Eq. (A.7). By that reason, because of zero two first moments of the collision term $\delta S t$ (A.7), there are no contributions from (A.7) into the continuity equation [zero $\mathbf{p}$ moment of the Boltzmann equation (8)] and, explicitly, into the momentum equation [the first $\mathbf{p}$ moment of (8)]. This term $\delta S t$ will affect only on the momentum flux tensor $\delta \Pi_{\mu \nu}$ (12) through the solutions (19) for the distribution function $\delta f$ [see, e.g., Eq. (13)] in terms of the viscosity coefficients [Eq. (16)].

For the integration over $\mathbf{p}_{1}$ in Eq. (A.7), it is convenient to use the system of the center of mass for a given two-body collision, with the symmetry $z$ axis directed along the relative motion of projectile particle having the reduced mass (Fig. 1). We transform the integral over 


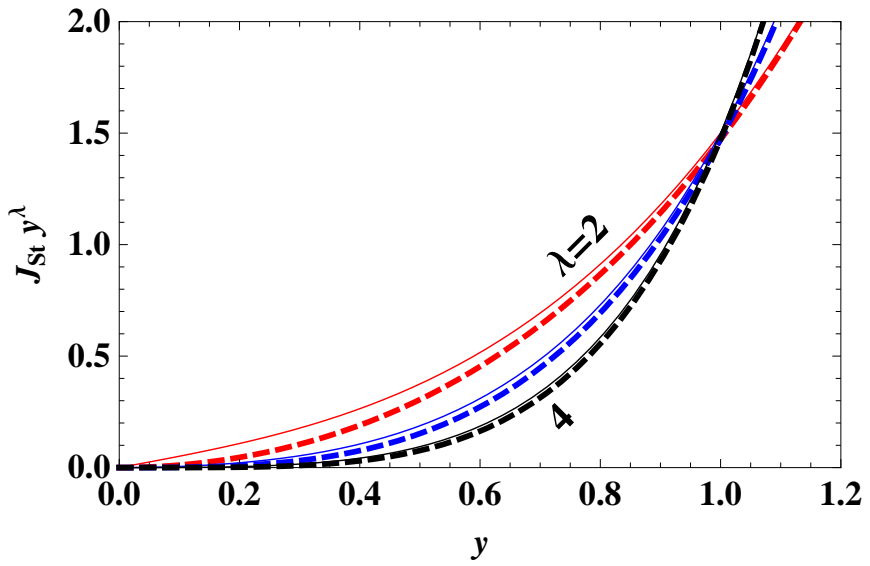

FIG. 5. Integral $y^{\lambda} \mathcal{J}_{S t}(y)$ [Eq. (A.13), solid] vs $y$ for powers $\lambda=2$ (red), 3 (blue), and 4 (black); dashed lines show those with the corresponding asymptotics [Eq. (A.15)] up to fourth order.

$\mathbf{p}_{1}$ to the relative momentum $\mathbf{q}=\mathbf{p}_{1}-\mathbf{p}$. Then, using the spherical-coordinate system with the symmetry $z$ axis directed along the vector $\mathbf{q}$ of the relative motion of projectile particle (Fig. 1), $\mathrm{d} \mathbf{q}=q^{2} \mathrm{~d} q \sin \theta_{q} \mathrm{~d} \theta_{q} \mathrm{~d} \varphi_{q}$ and $\left(x_{q}=\cos \theta_{q}\right)$, one finds from (A.7)

$$
\begin{aligned}
& \delta S t_{L} \equiv \int \mathrm{d} \Omega_{p} Y_{L 0}(\hat{p}) \delta S t(\mathbf{p}) \\
= & \frac{\pi^{2} d^{2}}{4 m} f_{0}(p) f_{0}(p) \sum_{\ell} \chi_{\ell} \sqrt{(2 L+1)(2 \ell+1)} \\
\times & \int_{-1}^{1} \mathrm{~d} x P_{L}(x) \int_{-1}^{1} \mathrm{~d} x_{q} \int_{0}^{\infty} q^{3} \mathrm{~d} q \exp \left[-\frac{q^{2}+2 p q x_{q}}{2 m T}\right] \\
\times & \int_{-1}^{1} \mathrm{~d} x^{\prime}\left[P_{\ell}\left(x_{1}^{\prime}\right)+P_{\ell}\left(x^{\prime}\right)-P_{\ell}\left(x_{1}\right)-P_{\ell}(x)\right] .
\end{aligned}
$$

Here, $P_{\ell}\left(\cos \theta_{p}\right)=(4 \pi /(2 \ell+1))^{1 / 2} Y_{\ell 0}(\hat{p})$ is the Legendre polynomial of $\ell$ order, and several transformations of the angle coordinates are performed:

$$
\begin{aligned}
x & =\cos \theta_{p} \quad x_{1}=\cos \theta_{p_{1}}, \\
x^{\prime} & =\cos \theta_{p^{\prime}} \quad x_{1}^{\prime}=\cos \theta_{p_{1}^{\prime}} .
\end{aligned}
$$

Note that the integration over the azimuthal angle of the relative momentum was taken from zero to $\pi$ [13]. For the integration over the modulus of the relative momentum $q$, for the fixed $x$ and $x^{\prime}$, one can change the angle variables to functions of the relative $x_{q}$ :

$$
\begin{aligned}
& x_{1}^{\prime}=x_{1}+x-x^{\prime}, \\
& x_{1}=z \sqrt{2 m T} x_{q} / p+x .
\end{aligned}
$$

For the fixed $x$ and $x^{\prime}$ we integrate first analytically over the variable $z=q / p_{T}$, where $p_{T}=\sqrt{2 m T}$, and then over $x_{q}$. Integrating then, e.g., the $\ell=2$ term, $\delta S t_{2}$ of Eq. (A.8), explicitly over remaining angles $x$ and $x^{\prime}$, one obtains

$$
\delta S t_{2}=\frac{5 \pi^{2} d^{2}}{2 m} p_{T}^{4} \chi_{2} f_{0}(p) I_{\mathrm{St}}(p)
$$

where

$$
I_{\mathrm{St}}(p)=f_{0}(p) \int_{-1}^{1} \mathrm{~d} x P_{2}(x) \int_{-1}^{1} \mathrm{~d} x^{\prime} \int_{-1}^{1} \mathrm{~d} x_{q}
$$

$$
\begin{aligned}
& \times \int_{0}^{\infty} z^{3} \mathrm{~d} z \exp \left[-\left(z^{2}+2 p x_{q} z / p_{T}\right)\right] \\
& \times\left[P_{2}\left(z \sqrt{2 m T} x_{q} / p+2 x-x^{\prime}\right)+P_{2}\left(x^{\prime}\right)\right. \\
& \left.-P_{2}\left(z \sqrt{2 m T} x_{q} / p+x\right)-P_{2}(x)\right] \\
& =\frac{2 n\left(2 p^{2}+p_{T}^{2}\right)}{5 \pi p p_{T}^{4}} \operatorname{erf}\left(\frac{p}{p_{T}}\right)+\frac{4 n}{5 \pi^{3 / 2} p_{T}^{3}} \\
& \times \exp \left(-\frac{p^{2}}{p_{T}^{2}}\right)=\frac{4 n}{5 \pi p_{T}^{3}} \mathcal{J}_{\mathrm{St}}\left(p / p_{T}\right),
\end{aligned}
$$

with the error function $\operatorname{erf}(y)=2 \int_{0}^{y} \mathrm{~d} z \exp \left(-z^{2}\right) / \sqrt{\pi}$,

$$
\begin{gathered}
\mathcal{J}_{\mathrm{St}}(y)=\frac{y^{2}+1 / 2}{y} \operatorname{erf}(y) \\
+\frac{1}{\sqrt{\pi}} \exp \left(-y^{2}\right), \quad y=p / p_{T} .
\end{gathered}
$$

To reduce the BVKE to the perturbation eigenvalue problem [Eqs. (21) and (22)] for the eigenfunctions $\varphi(\hat{p})$ and eigenvalues $c=\omega /\left(k v_{T}\right)$ as solutions of the linear homogeneous equations for $\varphi(\hat{p})$, and dispersion equation for $c$ (Appendix B), we may derive now the accurate constant (independent of $y$ ) approximations to the function $\mathcal{J}_{\mathrm{St}}(y)$ [Eqs. (A.13)]. Using these approximations, one obtains Eqs. (26) with (27) for the collision term $\delta S t$ (A.8). Indeed, we may note that for the derivation of such approximations the collision term $S t$ [Eq. (A.7)] can be considered through all of its $\mathbf{p}$ moments. They are integrals over the modulus $p$, which are taken up to the constant from the product of $\mathcal{J}_{\mathrm{St}}(p)$ [Eq. A.13] and the power $p^{\lambda}$ at $\lambda \geq 2$, in addition to the Maxwell distribution function $f_{0}(p)$,

$$
\int_{0}^{\infty} \mathrm{d} p p^{\lambda} \delta S t_{2} \propto \chi_{2} \int_{0}^{\infty} \mathrm{d} y y^{\lambda} \mathcal{J}_{\mathrm{St}}(y) f_{0}\left(y p_{T}\right) .
$$

Figure 5 shows a fast convergence of the product $y^{\lambda} \mathcal{J}_{\mathrm{St}}(y)$ [Eq. (A.13)] of the integrand in Eq. (A.14) to its asymptotics at large $y$ in powers of $1 / y$ taking enough many terms,

$$
\mathcal{J}_{\mathrm{St}}(y)=y+\frac{1}{2 y}+\mathcal{O}\left(\frac{1}{y^{4}}\right), \quad y \gg 1
$$

up to fourth-order terms for all $y$ values owing to the power factor $p^{\lambda}(\lambda \geq 2)$. Evaluating a smooth asymptotical function $\mathcal{J}_{\mathrm{St}}(y)$ (A.15) with respect to the Maxwell distribution function $f_{0}\left(y p_{T}\right)$ at the maximum contribution into the integrals (A.14) at $y \approx 1\left(p \approx p_{T}\right)$, one obtains approximately the damping rate $\nu$ of the collisional term (27):

$$
\nu=n v_{T} \sigma \mathcal{J}_{\mathrm{St}}(1) \approx \frac{3 \pi n v_{T} d^{2}}{2} .
$$

Note that the second exponent term in Eq. (A.13) for $\mathcal{J}_{\text {St }}$ was exactly canceled by the second term of the error function expansion, that leads to a good relative accuracy (about 6\%) after neglecting terms of the order of $1 / y^{4}$ in asymptotics (A.15).

This accuracy can be checked by comparison of (A.16) with calculations of the exact function $\mathcal{J}_{\mathrm{St}}(y)$, and its 
average $\left\langle\mathcal{J}_{\mathrm{St}}(y)\right\rangle_{\text {av }}$ over $y$ with the static distribution $f_{0}$ (3),

$$
\left\langle\mathcal{J}_{\mathrm{St}}(y)\right\rangle_{\mathrm{av}}=\frac{\int_{0}^{\infty} y^{2} \mathrm{~d} y \mathcal{J}_{\mathrm{St}}(y) f_{0}(y)}{\int_{0}^{\infty} y^{2} \mathrm{~d} y f_{0}(y)}=\sqrt{\frac{8}{\pi}} .
$$

Calculating $\nu$ traditionally $[13,19,27]$ through the averaged value (A.17) of the collision term [ or $\left.\mathcal{J}_{\mathrm{St}}(y)(\mathrm{A} .13)\right]$ over all momenta $\mathbf{p}$ (or $y$ ), one obtains

$$
\begin{aligned}
\nu & \approx\langle\nu\rangle_{\mathrm{av}}=n v_{T} \sigma\left\langle\mathcal{J}_{\mathrm{St}}(y)\right\rangle_{\mathrm{av}} \\
& \approx \sqrt{8 / \pi} \pi n v_{T} d^{2} .
\end{aligned}
$$

Thus, both approximations for $\nu$, Eqs. (27) and (A.18), are almost the same within a good relative precision mentioned above.

\section{Appendix B: DERIVATIONS OF DISPERSION EQUATION}

To derive the dispersion equation (29) for the ratio $c=\omega /\left(k v_{T}\right)$ with respect to $c$ in the $\mathrm{FC}$ regime, one may specify a small perturbation parameter $\epsilon$ [Eq. (23)] in perturbation expansion for $\varphi(\hat{p})$ [Eqs. (21) and (22)]. Then, in the $\mathrm{FC}$ regime (small $\epsilon$ ), one can truncate the expansion of $\varphi(\hat{p})(25)$ over spherical functions $Y_{\ell 0}(\hat{p})$ in the plane-wave distribution function $\delta f(19)$ at the quadrupole value of $\ell, \ell \leq 2$, because of a fast convergence of the sum (25) over $\ell$ [14]. Substituting the plane-wave solution (19) with the multipole expansion (25) for $\varphi(\hat{p})$ in $\delta f$ into the BVKE (8), after simple algebraic transformations, one finally arrives (within the same approximations used in Appendix A) at the following linear equations $(L=0,1,2, \ldots)$ for $\varphi_{\ell}$ :

$$
\sum_{\ell} \mathcal{A}_{L \ell}(c) \varphi_{\ell}=0, \quad c=\omega /\left(k v_{T}\right),
$$

where

$$
\begin{gathered}
\mathcal{A}_{L \ell}(c) \equiv c \delta_{L \ell}-C_{\ell 1 ; L}+\frac{\mathcal{F}}{\sqrt{3}} \delta_{L 1} \delta_{\ell 0} \\
+i \gamma \delta_{\ell L}\left(1-\delta_{\ell 0}\right)\left(1-\delta_{\ell 1}\right), \\
C_{\ell 1 ; L}=\sqrt{\frac{4 \pi}{3}} \int \mathrm{d} \Omega_{p} Y_{L 0}(\hat{p}) Y_{10}(\hat{p}) Y_{\ell 0}(\hat{p}) \\
=\sqrt{\frac{2 \ell+1}{2 L+1}}\left(C_{\ell 0,10}^{L 0}\right)^{2},
\end{gathered}
$$

$C_{\ell 0,10}^{L 0}$ is the Clebsh-Gordan coefficients [38], and $\gamma$ is given by Eq. (31) (Appendix A). We multiplied the BVKE (8) by $Y_{L 0}(\hat{p})$, and integrated term by term over angles $\mathrm{d} \Omega_{p}$ of the unit momentum vector $\hat{p}$ in the spherical coordinate system with the polar $z$ axis along the unit wave vector $\hat{k}$. The integrals can be calculated explicitly by using the orthogonal properties of spherical functions and Clebsh-Gordan techniques for calculations of a few spherical function products in the integrand. The matrix $\mathcal{A}_{L \ell}$ has a simple structure. At the diagonal, one finds non-zero values $\mathcal{A}_{\ell \ell}$ depending on the sound velocity $c$. There are also two $L=\ell \pm 1$ lines, parallel to the diagonal, above and below it, with the nonzero number coefficients, depending on the Clebsh-Gordan coefficients through Eq. (B.3). They are independent of the velocity $c$ and the dimensionless collisional rate $\gamma$. Other matrix elements are zero. The isotropic mean field $\delta U(9)$ influences, through the interaction constant $\mathcal{F}(30)$, on only one matrix element, $\mathcal{A}_{01}=\mathcal{F} / \sqrt{3}-C_{01,0}$. The damping rate constant $\gamma$ related to the collision integral [Eq. (26)] are placed only in the main diagonal $\mathcal{A}_{\ell \ell}$ at $\ell \geq 2, \mathcal{A}_{\ell \ell}=c+i \gamma$ because of the conservation conditions, as explained above (Appendix A). For the FC regime, because of large $\gamma$, one notes the convergence of the coefficients $\varphi_{\ell}$ of the expansion in multipolarities (25): Any $\varphi_{\ell}$ at $\ell \geq 2$ is smaller than $\varphi_{\ell-1}$ by factor $1 /(c+i \gamma)[14,17]$. See more explicit expressions for ratios of the amplitudes $\varphi_{\ell}$ in Appendix C [Eq. (C.8)] in the case of the quadrupole truncation of the characteristic matrix $\mathcal{A}$. Truncating this matrix at the quadrupole value $\ell \leq 2$ and $L \leq 2$, one obtains the following simple $3 \times 3$ matrix

$$
\mathcal{A}^{(2)}=\left(\begin{array}{ccc}
c & -C_{11 ; 0} & 0 \\
\mathcal{F} / \sqrt{3}-C_{01 ; 1} & c & -C_{21 ; 1} \\
0 & -C_{11 ; 2} & c+i \gamma
\end{array}\right),
$$

with $C_{01 ; 1}=C_{11 ; 0}=1 / \sqrt{3}$ and $C_{21 ; 1}=C_{11 ; 2}=$ $2 / \sqrt{15}$. Accounting for Eq. (31) for $\gamma$, and explicit expressions for these constants $C_{\ell 1 ; L}$ (B.3), in the quadrupole $\mathrm{FC}$ case, one obtains the condition of existence of nonzero solutions $\left[\operatorname{det} \mathcal{A}^{(2)}(c)=0\right.$ ] of linear equations (B.1), that is the cubic equation (29) with respect to $c$.

Substituting $c=c_{r}+i c_{i}$ into the dispersion equation (29), one can use the overdamped conditions within the FC regime,

$$
|c / \gamma|=|\omega \tau| \ll 1, \quad\left|c_{r} / c_{i}\right| \ll 1 .
$$

Then, at leading order one obtains (for $\gamma \neq 0$ )

$$
-i \mathcal{F}_{1} \frac{c_{i}}{\gamma}-\mathcal{F}_{1} \frac{c_{r}}{\gamma}-i \mathcal{F}_{2}=0,
$$

where $\mathcal{F}_{1}=3 / 5-\mathcal{F} / 3$ and $\mathcal{F}_{2}=(1-\mathcal{F}) / 3$, and $\gamma$ is given by Eq. (31). Separating real and imaginary parts, at leading order within the conditions (B.5), one finds the overdamped solution,

$$
c_{r}=0, \quad c_{i}=-\frac{\mathcal{F}_{2}}{\mathcal{F}_{1}} \gamma,
$$

that is identical to Eq. (33).

\section{Appendix C: MOMENTS OF THE DISTRIBUTION FUNCTION AND VISCOSITY}

For the shear viscosity $\eta$ [Eq. (16)], one has to calculate the matrices $\mathcal{U}_{\mu \nu}$ [Eq. (17)] and $\delta \sigma_{\mu \nu}$ [Eq. (13)]. Taking the polar axis of the spherical coordinate system in the momentum space along the unit wave vector $\hat{k}=\mathbf{k} / k$, we note that these matrices are symmetric with zero nondiagonal terms, and

$$
\mathcal{U}_{x x}=\mathcal{U}_{y y}=-\frac{1}{2} \mathcal{U}_{z z},
$$




$$
\sigma_{x x}=\sigma_{y y}=-\frac{1}{2} \sigma_{z z}
$$

We find easy these relations using the symmetry arguments and properties of the integrals of the plane-wave solution (19) for $\delta f$ over the angles $\mathrm{d} \Omega_{p}$ of vector $\mathbf{p}$. Therefore, from Eqs. (17), (13), (19) and (25) one has to obtain only the simplest $z z$ components,

$$
\begin{aligned}
\mathcal{U}_{z z} & =2 \frac{\partial u_{z}}{\partial z}-\frac{2}{3} \nabla \mathbf{u}=\frac{4}{3} i k \widetilde{u}_{z} \exp (-i \omega t+i \mathbf{k r}) \\
\sigma_{z z} & =\widetilde{\sigma}_{z z} \exp (-i \omega t+i \mathbf{k r})
\end{aligned}
$$

where

$$
\begin{aligned}
\widetilde{u}_{z} & =\int \frac{\mathrm{d} \mathbf{p}}{n m} p_{z} f_{0}(p) \varphi(\hat{p})=\frac{v_{T}}{\pi \sqrt{3}} \varphi_{1} \\
\varphi_{\ell} & =\int \mathrm{d} \Omega_{p} Y_{\ell 0}(\hat{p}) \varphi(\hat{p})
\end{aligned}
$$

and

$$
\begin{aligned}
& \widetilde{\sigma}_{z z}=-\int \frac{\mathrm{d} \mathbf{p}}{3 m}\left(3 p_{z}^{2}-p^{2}\right) f_{0}(p) \varphi(\hat{p}) \\
= & -\frac{2}{3 m} \sqrt{\frac{4 \pi}{5}} \int p^{4} \mathrm{~d} p f_{0}(p) \int \mathrm{d} \Omega_{p} Y_{20}(\hat{p}) \varphi(\hat{p}) \\
= & -\frac{n T}{\sqrt{5 \pi}} \varphi_{2} .
\end{aligned}
$$

We calculated explicitly the Gaussian-like integrals over $p$ using the static distribution function $f_{0}$ [Eq. (3)],

$$
I_{\lambda}=\int_{0}^{\infty} \mathrm{d} p p^{\lambda} f_{0}(p)=\frac{n p_{T}^{\lambda-2}}{2 \pi^{3 / 2}} \Gamma\left(\frac{\lambda+1}{2}\right),
$$

where $\Gamma(x)$ is the $\Gamma$ function. Using the orthogonal properties of the spherical functions and Eqs. (C.3), (C.4) and (C.5), from Eq. (16), one arrives at

$$
\eta=\frac{9 i \sqrt{\pi}}{4 \sqrt{15}} \frac{n T c}{\omega} \frac{\varphi_{2}}{\varphi_{1}} .
$$

So far we did not use a specific regime of collisions and the truncated linear system of equations (B.1). Solving these equations (B.1), one obtains

$$
\begin{aligned}
& \frac{\varphi_{0}}{\varphi_{1}}=\frac{1}{\sqrt{3} c}, \\
& \frac{\varphi_{2}}{\varphi_{1}}=\frac{2}{\sqrt{15}(c+i \gamma)} .
\end{aligned}
$$

With these expressions, from Eq. (C.7) one obtains

$$
\begin{aligned}
\eta & =\frac{3 \sqrt{\pi}}{10} \frac{1}{1+c /(i \gamma)} \frac{n T}{\nu} \\
& =\frac{1}{5 \sqrt{2 \pi}} \frac{1}{1+c /(i \gamma)} \frac{\sqrt{m T}}{d^{2}} .
\end{aligned}
$$

Substituting the overdamped solution for the sound velocity [Eq. (33)], from Eq. (C.9) one obtains Eq. (36).
[1] L.P. Csernai, J.I. Kapusta, and L.D. McLerran, Phys. Rev. Lett. 97, 152303 (2006).

[2] C. Chafin and T. Schaefer, Phys. Rev. A 87, 023629 (2013).

[3] A. Wiranata and M. Prakash, Phys. Rev. C 85, 054908 (2012).

[4] M. Albright and J.I. Kapusta, Phys. Rev. C 93, 014903 (2016).

[5] S. Chapman and T.G. Cowling, The Mathematical Theory of Non-uniform Gases, (Cambridge University Press, Cambridge, UK, 1952).

[6] G.E. Uhlenbeck, G.W. Ford, Lectures in statistical mechanics in Lectures in Applied Mathematics, Proceedings of the Summer Seminar, Boulder, Colorado,1960, Vol. I (Americal Mathematical Society, Providence, RI, 1963).

[7] K. Huang, Statistical Mechanics ( Wiley \& Sons, New York, 1963; 2nd ed., 1987).

[8] V.P. Silin, Introduction to the Kinetic Theory of Gases (Nauka, Moscow, 1971).

[9] J.H. Fertziger and H.G. Kaper, Mathematical Theory of Transport Processes in Gases, (North-Holland, Amsterdam, 1972).

[10] E.M. Lifshitz and L.P. Pitajevski, Physical Kinetics, Course of Theoretical Physics Vol. 10 (Nauka, Moscow, 1981).

[11] M. Prakash, M. Prakash, R. Venugopalan, and G. Welke, Phys. Rep. 227, 331 (1993).

[12] M.I. Gorenstein, M. Hauer, and O.M. Moroz, Phys. Rev.
C 77, 024911 (2008).

[13] A.A. Abrikosov and I.M. Khalatnikov, Rep. Prog. Phys. 22, 329 (1959).

[14] J. Sykes and G.A. Brooker, Ann. Phys. (NY) 56, 1 (1970); G.A. Brooker and J. Sykes, ibid. 61, 387 (1970).

[15] R. Balescu. Equilibrium and Non-equilibrium Statistical Mechanics (Wiley-Interscience, New York, 1975).

[16] G. Baym and C.J. Pethick, Landau Fermi Liquid Theory (Wiley \& Sons New York, 1991).

[17] V.M. Kolomietz, A.G. Magner, and V.A. Plujko, Z. Phys., 345, 131 (1993); 345137 (1993).

[18] A.G. Magner, V.M. Kolomietz, H. Hofmann, and S. Shlomo, Phys. Rev. C 51, 2457 (1995).

[19] C.J. Pethick and H. Smith, Bose-Einstein Condensation in Dilute Gases, (Cambridge University Press, Cambridge, UK, 2002).

[20] V.M. Kolomietz, V.A. Plujko, and S. Shlomo, Phys. Rev. C 54, 3014 (1996).

[21] V.M. Kolomietz, S.V. Lukyanov, V.A. Plujko, and S. Shlomo, Phys. Rev. C 58, 198 (1998).

[22] V.A. Plujko, Acta Phys. Pol. B 30, 1383 (1999).

[23] V.A. Plujko, O.M. Gorbachenko, and M.O. Kavatsyuk, Acta Phys. Slov. 51, 231 (2001).

[24] V.A. Plujko, S.N. Ezhov, O.M. Gorbachenko, and M.O. Kavatsyuk, J.Phys: Cond.Mater. 14, 9473 (2002).

[25] V.M. Kolomietz and S. Shlomo, Phys. Rep.390, 133 (2004).

[26] E.A. Spiegel and J.-L. Thiffeault, Phys. Fluids, 15, 3558 (2003). 
[27] P. Massignan, G.M. Bruun, and H. Smith, Phys. Rev. A 71, 033607 (2005).

[28] A.G. Magner, D.V. Gorpinchenko, and J. Bartel, Phys. At. Nucl., 77, 1229 (2014).

[29] L.D. Landau and E.M. Lifshitz, Statistical Physics, Course of Theoretical Physics Vol. 5 (Pergamon, Oxford, UK, 1975).

[30] V. Vovchenko, D.V. Anchishkin, and M.I. Gorenstein, Phys. Rev. C 91, 064314 (2015).

[31] L.D. Landau and E.M. Lifshitz, Hydrodynamics, Course of Theoretical Physics Vol. 6 (Nauka, Moscow, 2000).

[32] H. Hofmann, The Physics of Warm Nuclei with Analogies to Mesoscopic Systems (Oxford University Press, Oxford, UK, 2008).

[33] N. Demir, A. Wiranata, J. Phys.: Conf. Ser. 535, 012018
(2014).

[34] E. Madelung, Mathematical Methods in Physics (Fizmatgiz, Moscow, 1961).

[35] A.G. Magner, D.V. Gorpinchenko, and J. Bartel, arXiv:1604.06866 [nucl-th] [Phys. At. Nucl. (to be published)] (2016).

[36] A.B. Bhatia, Ultrasonic Absorption: An Introduction to the Theory and Dispersion in Gases, Liquids and Solids (Dover, New York, 1985).

[37] X. Chen, H. Rao, and E.A. Spiegel, Phys. Lett. A 271, 87 (2000); Phys. Rev. E 64, 046308 (2001).

[38] D.A. Varshalovich, A.N. Moskalev, and V.K. Khersonsky, Quantum Theory of Quantum Angular Momentum (World Scientific, New York, 1988) . 